\title{
Seletividade de associações e doses de herbicidas em pós emergência do trigo
}

\author{
Selectivity of associations and rates of the herbicides in \\ wheat post emergence
}

\section{Cristiano Piasecki ${ }^{1 *}$, Marcos Ivan Bilibio ${ }^{2}$, Henrique Fries ${ }^{3}$, Joanei Cechin $^{4}$, Maicon Fernando Schmitz ${ }^{4}$, Jonas Rodrigo Henckes ${ }^{4}$, Juliano Gazola ${ }^{4}$}

Resumo - As principais plantas daninhas encontradas na cultura do trigo são o nabo e o azevém, e seu controle em pós-emergência é baseado em herbicidas inibidores da enzima acetolactato sintase (ALS) e acetil coenzima A carboxilase (ACCase) que, em função da resistência, limita as opções de controle. Associações de herbicidas têm sido utilizadas em pós-emergência do trigo, porém, seus efeitos na cultura são pouco conhecidos. O objetivo deste trabalho foi avaliar a seletividade de associações de herbicidas e doses aplicados em pós-emergência do trigo. Foram realizados três experimentos a campo em sistema de semeadura direta, no delineamento de blocos ao acaso com três repetições. Os experimentos foram conduzidos nos municípios de Santa Bárbara do Sul-RS, Cruz Alta-RS e Tupanciretã-RS. Foram estudadas doses e associações entre os herbicidas 2,4-D, metsulfuron-methyl, iodosulfuron, piroxsulam e saflufenacil aplicados no perfilhamento do trigo. As variáveis avaliadas foram fitotoxicidade aos sete, 14, 21 e 35 dias após a aplicação (DAA), rendimento de grãos $\left(\mathrm{kg} \mathrm{ha}^{-1}\right)$ e peso hectolitro dos grãos de trigo $(\mathrm{PH})$, sendo os dados dos três experimentos analisados de forma conjunta. Aos 35 DAA as maiores fitotoxicidades foram observadas para metsulfuron-methyl na dose de 9 g i.a. ha ${ }^{-1}$ e para saflufenacil associado ao metsulfuron-methyl. Os resultados de rendimento de grãos demonstraram que a associação entre 2,4-D e metsulfuron-methyl, saflufenacil isolado ou associado à metsulfuron-methyl $\mathrm{e} o$ iodosulfuron-methyl foram seletivos para o trigo. $\mathrm{O}$ aumento da dose de metsulfuron-methyl ( 6 e $9 \mathrm{~g}$ i.a. ha $^{-1}$ ) e a dose de registro de piroxsulam causaram redução significativa na rendimento de grãos de grãos de trigo na média dos três experimentos. $\mathrm{O} \mathrm{PH}$ dos grãos de trigo não foi influenciado pelos herbicidas estudados.

Palavras-chave: Triticum aestivum, mistura de herbicidas, mistura em tanque, resistência

Abstract - The main weeds in wheat are wild radish and ryegrass, and its post-emergence control is based on herbicides inhibiting the enzyme acetolactate synthase (ALS) and acetyl coenzyme A carboxase (ACCase), which as a function of resistance limits the control options. Herbicide combinations have been used for post-emergence of wheat; however, the effects of herbicidal combinations are poorly understood. The objective of this work was to evaluate the selectivity of

Recebido: Agosto 17, 2017. Aceito: Fevereiro 06, 2018.

${ }^{1}$ Programa de Pós-graduação em Fitossanidade, Departamento de Fitossanidade, Universidade Federal de Pelotas UFPel, Campus Universitário, Capão do Leão, CEP 96010-900, Pelotas, RS, Brasil. E-mail: c_piasecki@hotmail.com

${ }^{2}$ Programa de Pós-graduação em Agronomia, Universidade de Passo Fundo - UPF, Passo Fundo, RS, Brasil. E-mail: marcosbilibio@yahoo.com.br

${ }^{3}$ Universidade Federal de Santa Maria - UFSM, Santa Maria, RS, Brasil. E-mail: henrique_fries@hotmail.com

${ }^{4}$ Programa de Pós-graduação em Fitossanidade/Herbologia, Universidade Federal de Pelotas - UFPel, Pelotas, RS, Brasil.E-mail: joaneicechin@yahoo.com.br; maicon_schmitz@hotmail.com; jhenckes@hotmail.com; julianogazola@hotmail.com 
wheat to herbicide associations and rates applied in post-emergence. Three field experiments were carried out in a no-tillage system, in a randomized complete block design with three replicates. The experiments were conducted in Santa Bárbara do Sul-RS, Cruz Alta-RS and Tupanciretã-RS. Rates and associations between the 2,4-D herbicides, metsulfuron-methyl, iodosulfuron, pyroxsulam and saflufenacil applied in wheat tillering were studied. The evaluated variables were phytotoxicity at seven, 14, 21 and 35 days after application (DAA), yield $\left(\mathrm{kg} \mathrm{ha}^{-1}\right)$ and hectoliter weigh (HW). The data were analyzed together. At 35 DAA, the highest phytotoxicities were observed for metsulfuron-methyl at the dose of $9 \mathrm{~g}$ a.i. ha ${ }^{-1}$ and for saflufenacil associated with metsulfuron-methyl. The results of yield demonstrate that the association between 2,4-D and metsulfuron-methyl, saflufenacil isolated or associated with metsulfuron-methyl, and iodosulfuron are selective for wheat. Metsulfuron-methyl at rates of 6 and $9 \mathrm{~g}$ a.i. ha ${ }^{-1}$ and pyroxsulam caused a significant reduction in wheat yield and were not considered selective. However, the hectoliter weight of the grains was not influenced by herbicides used of this study.

Keywords: Triticum aestivum, herbicide mix, tank mix, resistance

\section{Introdução}

O trigo (Triticum aestivum L.) é um dos principais cereais cultivados no mundo com produção superior a 700 milhões de toneladas anualmente e, considerada a base alimentar para humanos. No Brasil, o trigo é cultivado em cerca de dois milhões de hectares com predominância na região Sul, onde a produção é de em torno de $50 \%$ da demanda nacional (CONAB, 2017). Vários fatores bióticos e abióticos afetam a rendimento de grãos da cultura, dentre os bióticos as plantas daninhas, são as principais responsáveis pela redução da qualidade e quantidade do produto colhido (Vargas e Roman, 2005).

A aplicação de herbicidas tem sido o principal método de controle de plantas daninhas na cultura do trigo, no entanto, a intensiva e a inadequada aplicação de herbicidas selecionaram plantas daninhas resistentes (Mariani et al., 2016). As principais plantas daninhas em lavouras de trigo no Sul do Brasil são nabo (Raphanus spp.) resistente a herbicidas inibidores da enzima acetolactato sintase (ALS), e azevém (Lolium multiflorum) resistente aos inibidores das enzimas 5-enolpiruvilchiquimato-3-fosfato sintase (EPSPs), acetil coenzima A carboxilase (ACCase) e ALS (Heap, 2017). No entanto, os inibidores da ALS têm sido os principais herbicidas empregados no controle de azevém e nabo (Geier et al., 2011), que, na maioria das vezes, ocorrem simultaneamente nas áreas de cultivo.

A maior dificuldade no controle de plantas daninhas em trigo é verificada em pós emergência, pois poucos herbicidas possuem registro para esta modalidade de aplicação e, além disso, para a maior parte dos herbicidas registrados, as principais plantas daninhas apresentam resistência (Mariani et al., 2016; Heap, 2017; Brasil, 2017). No Brasil os herbicidas registrados para aplicação em pós-emergência do trigo são bentazona, clodinafope+propargil, diclofop-mehtyl, iodosulfuron-methyl, metribuzin, metsulfuron-methyl e 2,4-D (Brasil, 2017). Embora saflufenacil cause injúrias, muitos agricultores norte americanos preferem aplica-lo em pós-emergência do trigo de primavera junto a aplicação de nitrogênio (Frihauf et al., 2010a). Apesar das injúrias causadas, o saflufenacil pode ser alternativa para o controle de eudicotiledôneas em pós-emergência do trigo, especialmente para o controle de nabo resistente a ALS (Frihauf et al., 2010b).

A dificuldade no controle em função da resistência e a falta de novos herbicidas com mecanismos de ação diferenciados e com registro para aplicação em pós-emergência, têm obrigado triticultores a buscarem alternativas. Dessa forma, a aplicação 
de misturas de herbicidas em tanque tem sido a forma encontrada para se obter adequado controle das plantas daninhas. Entretanto, a seletividade dos principais herbicidas na cultura deve ser considerada, uma vez que, a mistura em tanque pode resultar em efeitos sinérgicos ou antagônicos, que podem comprometer o controle e a rendimento de grãos da cultura (Singh et al., 2011). Por outro lado, poucos estudos demonstram os efeitos de fitotoxicidade de associações entre herbicidas e doses em pós-emergência do trigo. Assim, o objetivo deste trabalho foi avaliar a seletividade ao trigo de associações e doses de herbicidas aplicados em pós-emergência.

\section{Material e métodos}

Foram realizados três experimentos no ano de 2015 em três municípios no Rio Grande do Sul: Santa Bárbara do Sul, Cruz Alta e Tupanciretã (Tabelas 1 e 2). Os experimentos foram instalados no sistema de semeadura direta, em áreas com restos culturais de aveia-preta e azevém controlados 30 dias antes da semeadura (DAS) com clethodim 76,2 g i.a ha-1 e glyphosate 1080 g de e.a. ha ${ }^{-1}$. Logo após a semeadura foi realizada aplicação de $400 \mathrm{~g}^{\text {i.a ha }}{ }^{-1}$ de paraquat com objetivo de eliminar plantas remanescentes ou emergidas após a aplicação anterior de herbicidas. As três áreas apresentavam histórico de baixa infestação por plantas daninhas.

Nos três experimentos, o delineamento experimental utilizado foi o de blocos ao acaso com três repetições, em unidades experimentais de $10 \mathrm{~m}^{2}(2 \times 5 \mathrm{~m})$. O trigo cultivar Iguaçu foi semeado em espaçamento entrelinhas de $0,17 \mathrm{~m}$. Nos três locais, a adubação utilizada foi de $350 \mathrm{~kg} \mathrm{ha}^{-1}$ do fertilizante NPK 10-20-20, e $220 \mathrm{~kg} \mathrm{ha}^{-1}$ de uréia em cobertura aplicada 10 dias após a aplicação dos herbicidas, seguindo a interpretação das análises de solo (Tabela 2). Os demais tratos culturais foram realizados conforme as recomendações técnicas para a cultura do trigo (EMBRAPA, 2014).

$\mathrm{Na}$ Tabela 3 estão descritos os tratamentos utilizados nos três experimentos. As aplicações dos tratamentos foram realizadas no perfilhamento pleno do trigo (Tabela 1), sendo realizadas com pulverizador costal pressurizado à $\mathrm{CO}_{2}$, utilizando pontas do tipo leque 110.02 , espaçadas em $50 \mathrm{~cm}$, sendo o equipamento calibrado para pulverizar um volume de calda de $150 \mathrm{~L} \mathrm{ha}^{-1}$. No momento da aplicação, as áreas de trigo não apresentavam infestação de plantas daninhas. As condições ambientais durante a condução dos experimentos nos três locais estão apresentadas na Figura 1.

Tabela 1. Localização dos experimentos, datas de semeadura, emergência e aplicações dos tratamentos herbicidas.

\begin{tabular}{lcccccc}
\hline \multicolumn{1}{c}{ Município } & Latitude & Longitude & Semeadura & Emergência & Aplicação & DAE $^{1}$ \\
\hline Santa Bárbara do Sul & $2^{\circ} 23^{\prime} 14^{\prime \prime} \mathrm{S}$ & $5^{\circ} 15^{\prime} 04^{\prime \prime} \mathrm{O}$ & $17 / 06 / 2015$ & $26 / 06 / 2015$ & $06 / 08 / 2015$ & 41,0 \\
Cruz Alta & $2^{\circ} 38^{\prime} 13^{\prime \prime} \mathrm{S}$ & $53^{\circ} 32^{\prime} 12^{\prime \prime} \mathrm{O}$ & $19 / 06 / 2015$ & $25 / 06 / 2015$ & $05 / 08 / 2015$ & 41,0 \\
Tupanciretã & $2^{\circ} 00^{\prime} 22^{\prime \prime} \mathrm{S}$ & $53^{\circ} 38^{\prime} 38^{\prime \prime} \mathrm{O}$ & $19 / 06 / 2015$ & $25 / 06 / 2015$ & $08 / 08 / 2015$ & 44,0 \\
\hline
\end{tabular}

${ }^{1}$ Dias após a emergência.

Tabela 2. Informações da análise de solo referentes as três áreas onde os experimentos foram conduzidos.

\begin{tabular}{ccccccc}
\hline Área & Município/UF & Argila (\%) & $\mathbf{p H}_{\text {agua }}$ & M.O. (\%) & $\mathbf{C T C}_{\text {efetiva }} \mathbf{2}^{\mathbf{1}}$ & $\mathbf{V ~ ( \% ) ) ^ { \mathbf { 3 } }}$ \\
\hline 1 & Santa Bárbara do Sul & 38 & 5,9 & 2,1 & 5,2 & 59 \\
2 & Cruz Alta & 34 & 5,8 & 3,5 & 11,8 & 70,5 \\
3 & Tupanciretã & 15 & 6,2 & 2,3 & 5,3 & 78 \\
\hline
\end{tabular}

${ }^{1}$ Teor $(\%)$ de matéria orgânica do solo; ${ }^{2}$ Capacidade de troca de cátions $\left(\mathrm{cmol}_{\mathrm{c}} / \mathrm{dm}^{3}\right)$ determinada no $\mathrm{pH}$ do solo; ${ }^{3}$ Saturação por bases. 
Tabela 3. Tratamentos utilizados nos três experimentos.

\begin{tabular}{|c|c|c|c|c|c|}
\hline $\begin{array}{l}\text { Tratamento } \\
\text { número }\end{array}$ & Ingrediente ativo herbicida & $\begin{array}{c}\text { Dose } \\
\left(\mathrm{g} \mathrm{ha}^{-1}\right)^{5} \\
(\text { i.a. ou e.a. })\end{array}$ & $\begin{array}{c}\text { Nome comercial do } \\
\text { herbicida }\end{array}$ & $\begin{array}{l}\text { Formulação } \\
\text { herbicida }^{6}\end{array}$ & $\begin{array}{c}\text { Dose } \\
\left.(\text { p.c. ha- })^{-1}\right)^{7}\end{array}$ \\
\hline \multirow{3}{*}{1} & $2,4-\mathrm{D}$ & 670 & DMA 806 BR & $670 \mathrm{SL}^{1}$ & $1 \mathrm{~L}$ \\
\hline & Metsulfuron-methyl & 4,2 & Ally & $600 \mathrm{WG}^{3}$ & $7 \mathrm{~g}$ \\
\hline & Óleo Mineral & 320 & Nimbus & $428 \mathrm{EC}^{2}$ & $0,5 \% \mathrm{v} / \mathrm{v}$ \\
\hline \multirow{3}{*}{2} & $2,4-\mathrm{D}$ & 1.005 & DMA 806 BR & 806 BR SL & $1,5 \mathrm{~L}$ \\
\hline & Metsulfuron-methyl & 4,2 & Ally & $600 \mathrm{WG}$ & $7 \mathrm{~g}$ \\
\hline & Óleo Mineral & 320 & Nimbus & $428 \mathrm{EC}$ & $0,5 \% \mathrm{v} / \mathrm{v}$ \\
\hline \multirow{2}{*}{3} & $2,4-\mathrm{D}$ & 670 & DMA 806 BR & 806 BR SL & $1 \mathrm{~L}$ \\
\hline & Óleo Mineral & 320 & Nimbus & $428 \mathrm{EC}$ & $0,5 \% \mathrm{v} / \mathrm{v}$ \\
\hline \multirow{2}{*}{4} & 2,4-D & 1.005 & DMA 806 BR & 806 BR SL & $1,5 \mathrm{~L}$ \\
\hline & Óleo Mineral & 320 & Nimbus & $428 \mathrm{EC}$ & $0,5 \% \mathrm{v} / \mathrm{v}$ \\
\hline \multirow{2}{*}{5} & Metsulfuron-methyl & 4,2 & Ally & $600 \mathrm{WG}$ & $7 \mathrm{~g}$ \\
\hline & Óleo Mineral & 320 & Nimbus & $428 \mathrm{EC}$ & $0,5 \% \mathrm{v} / \mathrm{v}$ \\
\hline \multirow{2}{*}{6} & Metsulfuron-methyl & 6,0 & Ally & $600 \mathrm{WG}$ & $10 \mathrm{~g}$ \\
\hline & Óleo Mineral & 320 & Nimbus & $428 \mathrm{EC}$ & $0,5 \% \mathrm{v} / \mathrm{v}$ \\
\hline \multirow{2}{*}{7} & Metsulfuron-methyl & 9,0 & Ally & $600 \mathrm{WG}$ & $15 \mathrm{~g}$ \\
\hline & Óleo Mineral & 320 & Nimbus & $428 \mathrm{EC}$ & $0,5 \% \mathrm{v} / \mathrm{v}$ \\
\hline \multirow{2}{*}{8} & Iodosulfuron-methyl & 5,0 & Hussar & $50 \mathrm{WG}$ & $100 \mathrm{~g}$ \\
\hline & Ester Metílico de óleo de Soja & 320 & Aureo & $720 \mathrm{EC}$ & $0,3 \% \mathrm{v} / \mathrm{v}$ \\
\hline \multirow{2}{*}{9} & Piroxsulam & 18 & Tricea & $45 \mathrm{OD}^{4}$ & $0,4 \mathrm{~L}$ \\
\hline & Óleo Mineral & 570 & Joint Oil & $761 \mathrm{EC}$ & $0,5 \% \mathrm{v} / \mathrm{v}$ \\
\hline \multirow{2}{*}{10} & Saflufenacil & 42 & Heat & $700 \mathrm{WG}$ & $60 \mathrm{~g}$ \\
\hline & Óleo Mineral & 370 & Assist & $756 \mathrm{EC}$ & $0,5 \mathrm{~L}$ \\
\hline \multirow{3}{*}{11} & Saflufenacil & 42 & Heat & $700 \mathrm{WG}$ & $60 \mathrm{~g}$ \\
\hline & Metsulfuron-methyl & 4,2 & Ally & $600 \mathrm{WG}$ & $7 \mathrm{~g}$ \\
\hline & Óleo Mineral & 64 & Assist & $756 \mathrm{EC}$ & $0,5 \mathrm{~L}$ \\
\hline 12 & Testemunha sem herbicida & - & - & - & - \\
\hline
\end{tabular}

As variáveis avaliadas foram fitotoxicidade no trigo aos sete, 14, 21 e 35 dias após a aplicação (DAA), rendimento de grãos do trigo e peso do hectolitro dos grãos do trigo $(\mathrm{PH})$. Para a avaliação de fitotoxicidade foi utilizada a escala percentual visual onde, zero refere-se a ausência de fitotoxicidade e 100 refere-se a morte das plantas (SBCPD, 1995). Para determinar o rendimento de grãos do trigo foram colhidas seis linhas centrais de cada unidade experimental por três metros de comprimento $\left(3,06 \mathrm{~m}^{2}\right)$. Após a colheita, o material foi trilhado, pesado e determinada a umidade dos grãos. Após realizada a correção da umidade para
$13 \%$ foi determinado o rendimento de grãos por hectare e o PH.

Os dados das variáveis estudadas foram analisados quanto a normalidade pelo teste de Shapiro-Wilk e à homocedasticidade pelo teste de Hartley. Primeiramente os dados de cada experimento foram submetidos a análise de variância (ANOVA) individual pelo $\mathrm{F}$ teste a $5 \%$ de probabilidade. Após, foi realizada ANOVA conjunta dos três experimentos conforme descrito por Banzatto e Kronka (2006) a 5\% de probabilidade no teste F. A partir dos resultados da ANOVA conjunta, se verificada interação entre os fatores (tratamentos $x$ locais), procedeu-se a comparação entre as médias 

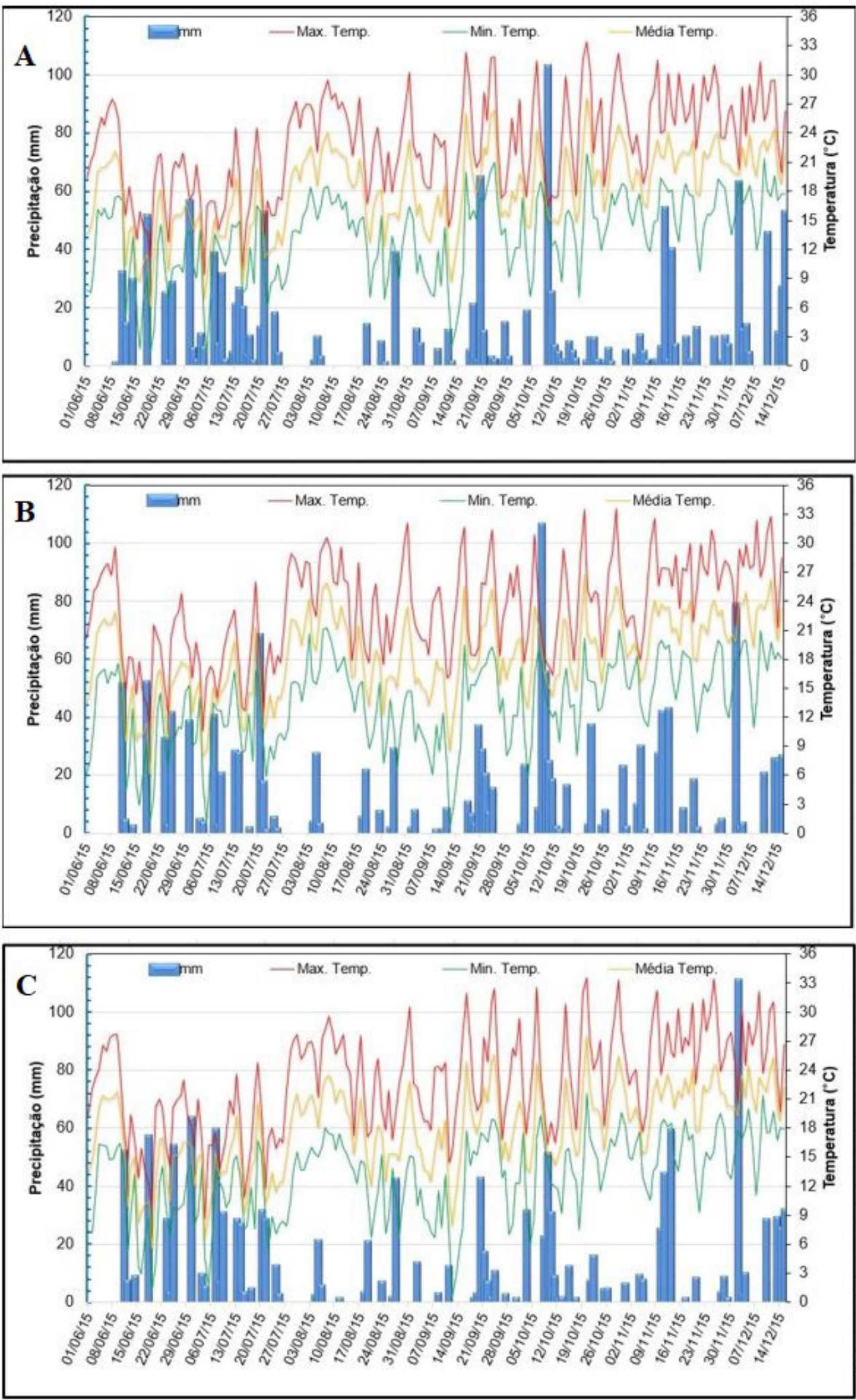

Figura 1. Dados meteorológicos para os locais de condução dos experimentos. (A) Santa Bárbara do Sul - RS; (B) Tupanciretã - RS; (C) Cruz Alta - RS. Fonte: SOMAR Meteorologia. 
dos tratamentos dentro de cada experimento (local) pelo teste de Scott-Knott $(\mathrm{p} \leq 0,05)$. No caso de interação não significativa, as médias gerais dos experimentos foram comparadas pelo teste de Scott-Knott $(\mathrm{p} \leq 0,05)$. Os resultados da rendimento de grãos foram considerados para determinar se o tratamento foi seletivo ou não para o trigo.

\section{Resultados e discussão}

A análise dos resultados obtidos nos experimentos evidenciou não haver necessidade de transformação dos dados, com base nos testes de Shapiro-Wilk e de Hartley. A análise de variância conjunta indicou efeito significativo entre os tratamentos e locais, e também para a interação entre tratamentos e locais para fitotoxicidade aos sete, 14, 21, 35 dias após a aplicação (DAA). Não houve efeito significativo para o peso do hectolitro $(\mathrm{PH})$ dos grãos de trigo em função dos tratamentos nos locais avaliados (Tabela 4). Para o rendimento de grãos do trigo a análise de variância conjunta não demonstrou interação entre tratamentos e locais. Dessa forma, foi calculada a média para cada tratamento dos três experimentos e as médias gerais dos tratamentos aplicados ao teste de comparação (Figura 2).

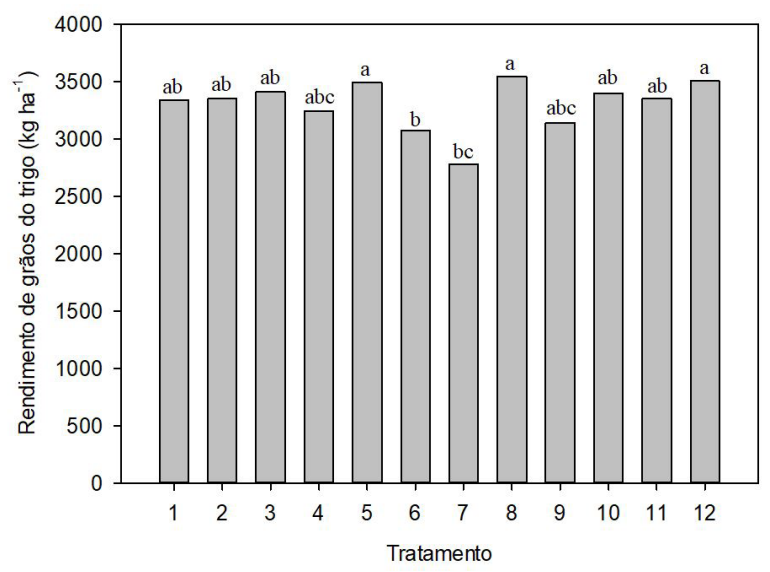

Figura 2. Rendimento médio de grãos de trigo em função da aplicação de herbicidas. Médias seguidas pelas mesmas letras não diferem pelo teste de Scott-Knott $(\mathrm{p} \leq 0,05)$. Resultado médio dos três experimentos.
Aos sete DAA, a aplicação de saflufenacil isolado na dose de $42 \mathrm{~g}$ i.a. ha $^{-1}$ e associado ao metsulfuron-methyl na dose de 4,2 g i.a. ha $^{-1}$ proporcionaram os maiores valores de fitotoxicidade que variaram de $13 \%$ a $22 \%$ nos diferentes locais avaliados e, foi similar a aplicação isolada de saflufenacil (Tabela 4). Para os demais herbicidas, os valores de fitotoxicidade foram todos abaixo de 13\%. Resultados semelhantes de seletividade na cultura do trigo foram observados para os herbicidas metsulfuron-methyl na dose de 2,4 e 3,6 g i.a. ha ${ }^{-1}$ e 2,4-D na dose de 480 e 960 g e.a. ha ${ }^{-1}$, iodosulfurom-metil e saflufenacil na dose de 5 e 49 gi.a. ha ${ }^{-1}$ e, piroxsulam na dose de $18 \mathrm{~g}$ i.a. ha ${ }^{-1}$ com fitotoxicidade abaixo de $10 \%$ aos sete DAA (Vargas e Roman, 2005; Geier et al., 2011).

No decorrer das avaliações realizadas aos 14, 21 e 35 DAA, a maior parte dos tratamentos apresentou redução da fitotoxicidade, exceto para o metsulfuron-methyl na dose $9 \mathrm{~g}$ i.a. ha $\mathrm{ha}^{-1} \mathrm{a}$ fitotoxicidade foi maior. Todavia, a aplicação de doses maiores de metsulfuron-methyl ocasionou aumento da fitotoxicidade no trigo e redução na capacidade de metabolização do herbicida (Tabela 4). Ao avaliar a seletividade de diferentes herbicidas em cereais de inverno, a fitotoxicidade em cevada foi de $15 \%$ para o herbicida metsulfuron-methyl na dose de 3,6 g i.a. ha-1 e ausente na dose de 2,4 g i.a. ha ${ }^{-1}$ aos 14 DAA (Vargas e Roman, 2005). Em trigo e cevada, o metabolismo do metsulfuron-methyl ocorre por hidroxilação seguido de conjugação com glicose ou, por hidroxilação do anel aromático (Anderson et al., 1989). Esse processo enzimático é mediado através de monooxigenação da citocromo $\mathrm{P} 450$ e por conjugação com glicosiltransferase, sendo o efeito estreitamente ligado a dose do herbicida, onde o excesso de produto pode inibir a enzima ALS e causar injúrias a cultura (Carvalho et al., 2009).

Para 2,4-D foi evidenciado baixa fitotoxicidade para aplicação isolada do herbicida independente da dose utilizada para os três locais de experimento com níveis inferiores a 5\% aos 14, 21 e 35 DAA, 
Tabela 4. Fitotoxicidade no trigo aos 7, 14, 21 e 35 dias após a aplicação (DAA) e peso do hectolitro dos grãos de trigo em função da aplicação dos herbicidas.

\begin{tabular}{|c|c|c|c|c|c|c|}
\hline \multirow{2}{*}{ Tratamento } & \multicolumn{6}{|c|}{ Santa Bárbara do Sul - RS } \\
\hline & Dose & 7 DAA & 14 DAA & 21 DAA & 35 DAA & PH \\
\hline 2,4-D + metsulfuron-methyl & $670+4,2$ & $3 \mathrm{~d}^{1}$ & $4 \mathrm{~b}$ & $1 \mathrm{c}$ & $1 \mathrm{~d}$ & $79^{\mathrm{ns}}$ \\
\hline $2,4-\mathrm{D}+$ metsulfuron-methyl & $1005+4,2$ & $5 \mathrm{c}$ & $4 \mathrm{~b}$ & $1 \mathrm{c}$ & $1 \mathrm{~d}$ & 81 \\
\hline $2,4-\mathrm{D}$ & 670 & $3 \mathrm{~d}$ & $2 \mathrm{~b}$ & $1 \mathrm{c}$ & $1 \mathrm{~d}$ & 81 \\
\hline $2,4-\mathrm{D}$ & 1005 & $5 \mathrm{c}$ & $3 \mathrm{~b}$ & $1 \mathrm{c}$ & $1 \mathrm{~d}$ & 80 \\
\hline Metsulfuron-methyl & 4,2 & $1 \mathrm{~d}$ & $3 \mathrm{~b}$ & $4 \mathrm{~b}$ & $1 \mathrm{~d}$ & 79 \\
\hline Metsulfuron-methyl & 6 & $4 \mathrm{c}$ & $3 \mathrm{~b}$ & $3 \mathrm{~b}$ & $1 \mathrm{~d}$ & 80 \\
\hline Metsulfuron-methyl & 9 & $7 \mathrm{c}$ & $10 \mathrm{a}$ & $8 \mathrm{a}$ & $13 \mathrm{a}$ & 79 \\
\hline Iodosulfuron-methyl & 5,0 & $4 \mathrm{c}$ & $10 \mathrm{a}$ & $9 \mathrm{a}$ & $4 \mathrm{c}$ & 79 \\
\hline Piroxsulam & 18 & $7 \mathrm{c}$ & $6 \mathrm{~b}$ & $4 \mathrm{~b}$ & $2 d$ & 78 \\
\hline Saflufenacil & 42 & $13 \mathrm{~b}$ & $12 \mathrm{a}$ & $10 \mathrm{a}$ & $9 \mathrm{~b}$ & 80 \\
\hline Saflufenacil + metsulfuron-methyl & $42+4,2$ & $17 \mathrm{a}$ & $13 \mathrm{a}$ & $9 \mathrm{a}$ & $12 \mathrm{a}$ & 79 \\
\hline Testemunha & --- & $0 \mathrm{~d}$ & $0 \mathrm{~b}$ & $0 \mathrm{c}$ & $0 \mathrm{~d}$ & 79 \\
\hline $\mathrm{CV}(\%)$ & & 32 & 28 & 27 & 32 & 2,1 \\
\hline Tratamento & \multicolumn{6}{|c|}{ Cruz Alta - RS } \\
\hline 2,4-D + metsulfuron-methyl & $670+4,2$ & $8 \mathrm{c}$ & $3 \mathrm{c}$ & $1 \mathrm{c}$ & $1 \mathrm{~d}$ & $79^{\text {ns }}$ \\
\hline 2,4-D + metsulfuron-methyl & $1005+4,2$ & $12 \mathrm{~b}$ & $6 \mathrm{c}$ & $4 \mathrm{~b}$ & $1 \mathrm{~d}$ & 81 \\
\hline $2,4-\mathrm{D}$ & 670 & $6 \mathrm{~d}$ & $5 \mathrm{c}$ & $1 \mathrm{c}$ & $1 \mathrm{~d}$ & 81 \\
\hline $2,4-\mathrm{D}$ & 1005 & $4 \mathrm{~d}$ & $4 \mathrm{c}$ & $1 \mathrm{c}$ & $1 \mathrm{~d}$ & 80 \\
\hline Metsulfuron-methyl & 4,2 & $4 \mathrm{~d}$ & $4 \mathrm{c}$ & $3 \mathrm{~b}$ & $3 \mathrm{c}$ & 79 \\
\hline Metsulfuron-methyl & 6 & $6 \mathrm{~d}$ & $4 \mathrm{c}$ & $4 \mathrm{~b}$ & $4 \mathrm{c}$ & 80 \\
\hline Metsulfuron-methyl & 9 & $13 \mathrm{~b}$ & $12 \mathrm{~b}$ & $18 \mathrm{a}$ & $18 \mathrm{a}$ & 79 \\
\hline Iodosulfuron-methyl & 5,0 & $9 \mathrm{c}$ & $6 \mathrm{c}$ & $4 \mathrm{~b}$ & $4 \mathrm{c}$ & 79 \\
\hline Piroxsulam & 18 & $9 \mathrm{c}$ & $4 \mathrm{c}$ & $4 \mathrm{~b}$ & $4 \mathrm{c}$ & 78 \\
\hline Saflufenacil & 42 & $22 \mathrm{a}$ & $22 \mathrm{a}$ & $18 \mathrm{a}$ & $15 \mathrm{~b}$ & 80 \\
\hline Saflufenacil + metsulfuron-methyl & $42+4,2$ & $22 \mathrm{a}$ & $20 \mathrm{a}$ & $17 \mathrm{a}$ & $18 \mathrm{a}$ & 79 \\
\hline Testemunha & --- & $0 \mathrm{e}$ & $0 \mathrm{c}$ & $0 \mathrm{c}$ & $0 \mathrm{~d}$ & 79 \\
\hline \multirow[t]{2}{*}{$\mathrm{CV}(\%)$} & & 22 & 27 & 27 & 23 & 2 \\
\hline & \multicolumn{6}{|c|}{ Tupanciretã - RS } \\
\hline 2,4-D + metsulfuron-methyl & $670+4,2$ & $3 \mathrm{~d}$ & $3 \mathrm{~d}$ & $1 \mathrm{c}$ & $1 \mathrm{~d}$ & $78^{\mathrm{ns}}$ \\
\hline 2,4-D + metsulfuron-methyl & $1005+4,2$ & $7 \mathrm{c}$ & $4 \mathrm{~d}$ & $1 \mathrm{c}$ & $1 \mathrm{~d}$ & 80 \\
\hline $2,4-\mathrm{D}$ & 670 & $6 \mathrm{c}$ & $4 \mathrm{~d}$ & $1 \mathrm{c}$ & $1 \mathrm{~d}$ & 80 \\
\hline $2,4-\mathrm{D}$ & 1005 & $10 \mathrm{~b}$ & $3 \mathrm{~d}$ & $4 \mathrm{c}$ & $1 \mathrm{~d}$ & 83 \\
\hline Metsulfuron-methyl & 4,2 & $1 \mathrm{~d}$ & $4 \mathrm{~d}$ & $1 \mathrm{c}$ & $1 \mathrm{~d}$ & 80 \\
\hline Metsulfuron-methyl & 6 & $11 \mathrm{~b}$ & $8 \mathrm{~d}$ & $1 \mathrm{c}$ & $1 \mathrm{~d}$ & 81 \\
\hline Metsulfuron-methyl & 9 & $12 \mathrm{~b}$ & $17 \mathrm{~b}$ & $17 \mathrm{a}$ & $18 \mathrm{a}$ & 80 \\
\hline Iodosulfuron-methyl & 5,0 & $12 \mathrm{~b}$ & $12 \mathrm{c}$ & $9 \mathrm{~b}$ & $5 \mathrm{c}$ & 80 \\
\hline Piroxsulam & 18 & $4 \mathrm{c}$ & $6 \mathrm{~d}$ & $3 \mathrm{c}$ & $1 \mathrm{~d}$ & 81 \\
\hline Saflufenacil & 42 & $18 \mathrm{a}$ & $23 \mathrm{a}$ & $17 \mathrm{a}$ & $14 \mathrm{~b}$ & 80 \\
\hline Saflufenacil + metsulfuron-methyl & $42+4,2$ & $22 \mathrm{a}$ & $15 \mathrm{~b}$ & $18 \mathrm{a}$ & $18 \mathrm{a}$ & 81 \\
\hline Testemunha & --- & $0 \mathrm{~d}$ & $0 \mathrm{~d}$ & $0 \mathrm{c}$ & $0 \mathrm{~d}$ & 82 \\
\hline $\mathrm{CV}(\%)$ & & 30 & 22 & 26 & 25 & 3,1 \\
\hline
\end{tabular}


não diferindo estatisticamente da testemunha sem aplicação (Tabela 4). Resultados semelhantes foram obtidos para aplicações de 2,4-D em doses inferiores a $960 \mathrm{~g}$ e.a. ha $\mathrm{h}^{-1}$ onde a fitotoxicidade foi menor que 5\% em cevada, aveia branca e azevém para todas as épocas avaliadas do estudo (Vargas e Roman, 2005). A tolerância de plantas ao herbicida 2,4-D envolve a translocação limitada via floema mediada por receptores de auxina (T1R1/AFB) responsáveis por repostas bioquímicas e transcricionais. Em plantas, a proteína T1R1 está envolvida na percepção do herbicida e no reconhecimento do substrato, além de atuar na regulação da síntese do etileno e do ácido abscísico através da expressão da enzima 9-cis-epoxicaroteno dioxigenase (NCED) no plastídio. Além disso, plantas tolerantes apresentam rápida hidroxilação da molécula pela $\mathrm{P} 450$ e conjugação com aminoácidos, tornando-as insensíveis ao herbicida (Grossmann, 2010; Mithila et al., 2011).

Ao avaliar a associação do metsulfuron-methyl na dose de 4,2 g i.a ha-1 com 2,4-D nas doses de 670 e 1005 g e.a. ha-1 , não foi observado aumento significativo na fitotoxicidade quando comparado a aplicação dos herbicidas isolados, o que demonstra que essa associação não causa elevada fitotoxicidade no trigo considerando as doses estudadas (Tabela 4). Estes resultados corroboram com os encontrados por Maciel et al. (2011) onde a aplicação de metsulfuron-methyl com 2,4-D na dose de 2,4 e $322,4 \mathrm{~g}$ ia. ha ${ }^{-1}$ na cultura do trigo resultou em baixos níveis de fitotoxicidade. A mistura é considerada uma importante ferramenta quando se deseja aumentar o espectro de controle, e também, para o manejo de biótipos resistentes de nabo ao metsulfuron-methyl que ocorrem na cultura do trigo (Baghestani et al., 2008).

A aplicação dos herbicidas piroxsulam e iodosulfurom demonstraram ser seletivos com níveis de fitotoxicidade aos 14 DAA inferiores a 6 e $12 \%$, respectivamente (Tabela 4). Resultados semelhantes foram encontrados para o herbicida piroxsulam aplicado no trigo em estádio de quatro folhas onde a fitotoxicidade foi menor que $10 \%$ na dose de 18,2 g i.a. ha-1 (Reddy et al., 2012). Em culturas tolerantes, a detoxificação dos herbicidas inibidores da acetolacto sintase (ALS) é considerado o principal mecanismo envolvido (Yu e Powles, 2014). Para o piroxsulam, a seletividade no trigo ocorre por absorção e translocação diferencial, além de rápido metabolismo pela $\mathrm{P} 450$ através de O-dealquilação do anel aromático na posição 5 do carbono (DeBoer et al., 2011).

Ao avaliar a aplicação do saflufenacil na dose de $42 \mathrm{~g}$ i.a. ha $\mathrm{h}^{-1}$ em pós emergência do trigo, os resultados evidenciaram que a fitotoxicidade variou de 12 a $23 \%$ nos três locais de estudo aos 14 DAA, com diminuição dos valores ao longo do período avaliado (Tabela 4). Alguns trabalhos reportam que o saflufenacil aplicado sobre o trigo pode apresentar níveis de fitotoxicidade variável dependendo da dose utilizada e, com níveis superiores a 20\% aos 14 DAA para doses superiores a 25 g i.a. ha-1 (Sikkema et al., 2008; Frihauf et al., 2010a). Outros trabalhos reportam que há diferenças na sensibilidade ao saflufenacil entre cultivares e estádios de aplicação em pós-emergência do trigo (Klein et al., 2006). Plantas tolerantes apresentam menor absorção e translocação da molécula e aumento na capacidade em detoxificação, levando a menor acúmulo de peróxido de hidrogênio e peroxidação lipídica (Grossmann et al., 2011). Além disso, ao avaliar a mistura em tanque do saflufenacil com metsulfuron-methyl nas doses estudadas, não houve diferença estatística comparada a aplicação isolada do saflufenacil, exceto para o estudo conduzido em Tupanciretã onde a fitotoxicidade foi $8 \%$ menor para a mistura aos 14 DAA (Tabela 4). Resultados similares foram obtidos para a mistura do saflufenacil com 2,4-D onde não houve aumento significativo da fitotoxicidade no trigo e, quando em mistura com bentazon, houve redução da fitotoxicidade em mais de $20 \%$ (Frihauf et al., 2010a). Outros estudos reportam resultados para a mistura de carfentrazone com metsulfuron-methyl na dose de 20 e 4 g i.a. ha-1, respectivamente, aplicado em pós-emergência do 
trigo a fim de aumentar o espectro de controle de plantas daninhas dicotiledôneas, onde os níveis de fitotoxicidade foram inferiores a $10 \%$ aos 10 DAA, sem ocasionar efeitos negativos na rendimento de grãos (Singh et al., 2011).

A rendimento de grãos média do trigo em todos os tratamentos foi de $3450 \mathrm{~kg} \mathrm{ha}^{-1}$, exceto para as aplicações de metsulfuron-methyl na dose de 6 e 9 g i.a. ha ${ }^{-1}$ onde houve decréscimo de 10 e $18 \%$ em relação a testemunha sem aplicação, respectivamente (Figura 2). Estes resultados corroboram com o efeito fitotóxico do herbicida metsulfuron-methyl quando ocorre aumento da dose na cultura do trigo onde a perda de rendimento de grãos foi superior a 11\% (Albrecht et al., 2010). Cabe ressaltar, que mesmo sendo evidenciados efeitos fitotóxicos da aplicação de saflufenacil isolado ou em mistura com o metsulfuron-methyl, não houve redução significativa da rendimento de grãos em relação a testemunha (Figura 2). Resultados semelhantes foram observados para a aplicação de saflufenacil em dose superior a $200 \mathrm{~g}$ i.a. ha ${ }^{-1}$ e superior a $40 \mathrm{~g}$ i.a. ha ${ }^{-1}$ em pré e pós emergência, respectivamente, onde houve redução da rendimento de grãos (Knezevic et al., 2010).

De forma geral dentre os locais e tratamentos em estudo, os resultados foram consistentes e oferecem importantes informações de aplicabilidade prática para os triticultores. Vale ressaltar a importância de ser observado o registro para cada herbicida e suas respectivas doses para aplicação em pós-emergência do trigo.

\section{Conclusões}

Os herbicidas 2,4-D (670 ou 1.005 g e.a. ha-1), metsulfuron-methyl (4,2 g i.a. ha-1), iodosulfuron, saflufenacil, bem como, as associações entre 2,4-D ou saflufenacil com metsulfuron-methyl (4,2 g i.a. ha $\left.{ }^{-1}\right)$ foram seletivas ao trigo. As injúrias causadas pela aplicação de piroxsulam e metsulfuron-methyl nas doses de 6 e $9 \mathrm{~g}$ i.a. ha ${ }^{-1}$ reduziram a rendimento de grãos da cultura. Os herbicidas, associações e doses estudados não influenciaram no PH dos grãos de trigo.

\section{Referências}

Albrecht, A.J.P.; Albrecht, L.P.; Migliavacca, R.A.; Reche, D.L.; Gasparotto, A.C.; Ávila, M.R. Metsulfuron-methyl no desempenho agronômico e na qualidade das sementes de trigo. Revista Brasileira de Herbicidas, v.9, n.2, p.54-62, 2010.

Anderson, J.J.; Priester, T.M.; Shalaby, L.M. Metabolism of metsulfuron methyl in wheat and barley. Journal of Agricultural and Food Chemistry, v.37, n.5, p.1429-1434, 1989.

Baghestani, M.A.; Zand, E.; Soufizadeh, S.; Beheshtian, M.; Haghighi, A.; Barjasteh, A.; et al. Study on the efficacy of weed control in wheat (Triticum aestivum L.) with tank mixtures of grass herbicides with broadleaved herbicides. Crop Protection, v.27, n.1, p.104-111, 2008.

Banzatto, D.A.; Kronka, S.N. Experimentação agrícola. 4.ed. Jaboticabal: Funep, 2006. 237p.

Brasil. Ministério da Agricultura, Pecuária e Abastecimento - MAPA. AGROFIT: sistema de agrotóxicos fitossanitários ou base de dados. Brasília: MAPA, 2017. Disponível em: < http:// agrofit.agricultura.gov.br/agrofit_cons/principal_ agrofit_cons $>$. Acesso em: ago. 2017.

Carvalho, S.J.P.; Nicolai, M.; Ferreira, R.R.; Figueira, A.V.O.; Christoffoleti, P.J. Herbicide selectivity by differential metabolism: considerations for reducing crop damages. Scientia Agrícola, v.66, n.1, p.136-142, 2009.

CONAB - Companhia Nacional de Abastecimento. Trigo: comparativo de área, rendimento de grãos e produção: safras 2016/2017. Brasília: CONAB, 2017. Disponível em: <http://www.conab.gov. br>. Acesso em: 25 jul. 2017.

DeBoer, G.J.; Thornburgh, S.; Gilbert, J.; Gast, R.E. The impact of uptake, translocation and metabolism on the differential selectivity between blackgrass and wheat for the herbicide pyroxsulam. Pest Management Science, v.67, n.3, p.279-286, 2011. 
EMBRAPA - Empresa Brasileira de Pesquisa Agropecuária. VIII Reunião da Comissão Brasileira de Pesquisa de Trigo e Triticale: informações técnicas para trigo e triticale: safra 2015. Brasília: EMBRAPA Trigo, 2014. Disponível em: $<$ https://www.embrapa.br/busca-de-publicacoes/-/ publicacao/1012773/informacoes-tecnicas-paratrigo-e-triticale---safra-2015>. Acesso em: abr. 2015.

Frihauf, J.C.; Stahlman, P.W.; Al-Khatib, K. Saflufenacil absorption and translocation in winter wheat (Triticum aestivum L.). Pesticide Biochemistry and Physiology, v.98, n.2, p.243247, $2010 b$.

Frihauf, J.C.; Stahlman, P.W.; Geier, P.W. Winter wheat and weed response to postemergence saflufenacil alone and in mixtures. Weed Technology, v.24, n.3, p.262-268, 2010a.

Geier, P.W.; Stahlman, P.W.; Peterson, D.E.; Claassen, M.M. Pyroxsulam compared with competitive standards for efficacy in winter wheat. Weed Technology, v.25, n.3, p.316-321, 2011.

Grossmann, K. Auxin herbicides: current status of mechanism and mode of action. Pest Management Science, v.66, n.2, p.113-120, 2010.

Grossmann, K.; Hutzler, J.; Caspar, G.; Kwiatkowski, J.; Brommer, C.L. Saflufenacil (KixorTM): biokinetic properties and mechanism of selectivity of a new protoporphyrinogen ix oxidase inhibiting herbicide. Weed Science, v.59, n.3, p.290-298, 2011.

Heap, I. The international survey of herbicide resistant weeds. 2017. Disponível em: <http:// www.weedscience.com/>. Acesso em: 15 jul. 2017.

Klein, R.N.; Martin, A.R.; Lyon, D.J. Annual broadleaf weed control in winter wheat. Nebraska: Lincoln Extension, University of Nebraska, 2006. Neb Guide G1241.

Knezevic, S.Z.; Datta, A.; Scott, J.; Charvat, L.D. Tolerance of winter wheat (Triticum aestivum L.) to pre-emergence and post-emergence application of saflufenacil. Crop Protection, v.29, n.2, p.148$152,2010$.
Maciel, C.G.D.; Moraes, D.W.; Balan, M.G. Associação de adjuvantes com herbicidas na dessecação e no controle em pósemergência de plantas daninhas na cultura do trigo. Revista Brasileira de Herbicidas, v.10, n.3, p.243-256, 2011.

Mariani, F.; Vargas, L.; Agostinetto, D.; Nohatto, M.A.; Langaro, A.C.; Duarte, T.V. Valor adaptativo e habilidade competitiva de azevém resistente e suscetível ao iodosulfuron em competição com o trigo. Pesquisa Agropecuária Brasileira, v.51, n.6, p.710-719, 2016.

Mithila, J.; Hall, J.C.; Johnson, W.G.; Kelley, K.B.; Riechers, D.E. Evolution of resistance to auxinic herbicides: Historical perspectives, mechanisms of resistance, and implications for broadleaf weed management in agronomic crops. Weed Science, v.59, n.4, p.445-457, 2011.

Reddy, S.S.; Stahlman, P.W.; Geier, P.W. Pyroxsulam and chlorpyrifos applied the same day injures wheat. Crop Management, v.11, n.1, 2012. http://dx.doi. org/10.1094/CM-2012-1211-01-RS.

SBCPD - Sociedade Brasileira da Ciência das Plantas Daninhas. Procedimentos para instalação, avaliação e análise de experimentos com herbicidas. Londrina: SBCPD, 1995. 42p.

Sikkema, P.H.; Shropshire, C.; Soltani, N. Tolerance of spring barley (Hordeum vulgare L.), oats (Avena sativa L.) and wheat (Triticum aestivum L.) to saflufenacil. Crop Protection, v.27, n.12, p.1495-1497, 2008.

Singh, S.; Punia, S.S.; Yadav, A.; Hooda, V.S. Evaluation of carfentrazone-ethyl+metsulfuronmethyl against broadleaf weeds of wheat. Indian Journal Weed Science, v.43, n.1, p.12-22, 2011.

Vargas, L.; Roman, E.S. Seletividade e eficiência de herbicidas em cereais de inverno. Revista Brasileira de Herbicidas, v.4, n.3, p.1-10, 2005.

Yu, Q.; Powles, S.B. Resistance to AHAS inhibitor herbicides: current understanding. Pest Management Science, v.70, n.9, p.1340-1350, 2014. 